\title{
Evaluation of Subfoveal Choroidal Thickness in Internal Carotid Artery Stenosis
}

\author{
Betül İlkay Sezgin Akçay, ${ }^{1}$ Esra Kardeş, ${ }^{1}$ Sultan Maçin, ${ }^{2}$ \\ Cihan Ünlü, ${ }^{1}$ Engin Bilge Özgürhan, ${ }^{3}$ Aydın Maçin, ${ }^{1}$ Tahir Kansu Bozkurt, ${ }^{1}$ \\ Ahmet Ergin, ${ }^{1}$ and Reyhan Surmeli ${ }^{4}$ \\ ${ }^{1}$ Ophthalmology Clinic, Ümraniye Research and Training Hospital, Istanbul, Turkey \\ ${ }^{2}$ Radiology Clinic, Ümraniye Research and Training Hospital, Istanbul, Turkey \\ ${ }^{3}$ Beyoğlu Research and Training Hospital, Ophthalmology Clinic, Beyoğlu Research and Training Hospital, Istanbul, Turkey \\ ${ }^{4}$ Neurology Clinic, Ümraniye Research and Training Hospital, Istanbul, Turkey
}

Correspondence should be addressed to Betül İlkay Sezgin Akçay; betul_sezgin@yahoo.com

Received 28 August 2015; Revised 5 January 2016; Accepted 11 January 2016

Academic Editor: Lawrence S. Morse

Copyright (C) 2016 Betül İlkay Sezgin Akçay et al. This is an open access article distributed under the Creative Commons Attribution License, which permits unrestricted use, distribution, and reproduction in any medium, provided the original work is properly cited.

Purpose. To evaluate the relationship between internal carotid artery (ICA) stenosis and subfoveal choroidal thickness (SFCT) in the elderly population. Methods. A total of 42 eyes of 21 patients with more than 70\% ICA stenosis (Group 1) on one side and less than $70 \%$ stenosis (Group 2) on the other side were recruited for this study. ICA stenosis was diagnosed using both the B-mode and Doppler ultrasound. The two groups were compared in terms of the percentage of stenosis, SFCT measurements, intraocular pressure, ocular perfusion pressure, refractive error, and peak systolic velocity. Eyes were examined with the RTVue-100 OCT device by the EDI-OCT technique. Results. The mean age of the patients was $71.9 \pm 10.8$ years. The mean percentage of ICA stenosis was $74 \pm 4.9 \%$ in Group 1 and $47.5 \pm 7.7 \%$ in Group 2. The mean SFCT was $231.9 \pm 44.6 \mu \mathrm{m}$ in Group 1 and $216.2 \pm 46.8 \mu \mathrm{m}$ in Group 2 , which was significantly lower $(P=0.028)$. A statistically significant positive correlation was found between the percentage of internal carotid artery stenosis and SFCT $(r=0896, P=0.001)$. Conclusions. Compensatory SFCT increase can be seen in ipsilateral internal carotid artery stenosis greater than $70 \%$.

\section{Introduction}

The choroid is a highly vascularized structure. Ninety-five percent of the blood flow into the eye supplies the uveal structures, and the choroid receives more than $70 \%$ of the portion that enters the uveal structures [1]. The ophthalmic artery, the first branch of the internal carotid artery (ICA), divides to form the central retinal artery and the posterior ciliary artery, which are responsible for the nourishment of the posterior choroid $[2,3]$. The choroid lies between the lamina fusca of the sclera and the retinal pigment epithelium [3]. The main function of the choroid is to provide oxygen and nourishment to the outer retinal layers and uveal structures [4].

The in vivo structure of the choroid cannot be visualized properly with conventional methods such as fundus photography and fluorescein angiography due to the pigments in the retinal pigment epithelium (RPE) that attenuate the incident light. Indocyanine green angiography allows better imaging of the choroidal vessels, but it does not provide cross-sectional data. Recently, Spaide et al. demonstrated that choroidal thickness could be measured successfully and noninvasively in vivo using commercially available spectraldomain optical coherence tomography (SD-OCT) devices that use the enhanced depth imaging (EDI) technique [5].

Severe stenosis of the extracranial segment of internal carotid artery (ICA) is the main reason for visual disturbances associated with ocular ischemia $[6,7]$. Acute transient monocular blindness due to entrapment of emboli in the retinal arterial system is the most common ischemic ocular symptom (30-40\%) [8]. Reported range of chronic progressive ocular ischemia in patients with carotid artery stenosis or occlusion is 5-21\% [9-12]. Extracranial carotid artery stenosis 
of more than $50 \%$ is present in 1.5 to $9 \%$ of the population, with a higher incidence in the elderly, and $70 \%$ stenosis of the ICA is the threshold currently used for surgical intervention $[13,14]$. An embolus originating in the carotid artery is likely to enter the ophthalmic artery, which is the first branch of the artery, leading to hypoperfusion of the retina and choroid. Choroidal thickness has attracted a great deal of interest in recent years to find out its association with many variables including age, sex, smoking, and disease states in recent studies [15-23]. However, extensive literature research did not reveal any information about effect of internal carotid artery stenosis on subfoveal choroidal thickness. Ocular ischemic syndrome is one of the most severe clinical entities associated with the carotid artery stenosis. Therefore, we aimed to evaluate whether the ICA stenosis of more than $70 \%$ has an effect on the ipsilateral subfoveal choroidal thickness before the onset of a symptomatic ischemic process by comparing to the opposite eye.

\section{Methods}

In this study, twenty-one patients with a diagnosis of ICA stenosis greater than $70 \%$ on only one side (the opposite ICAs had less than $70 \%$ stenosis) were included at the Radiology Clinic of the Ümraniye Research and Training Hospital between November 2013 and November 2014. The eyes were divided into two groups with respect to the degree of stenosis. The eyes ipsilateral to the ICA with greater stenosis (>70\%) were recruited as Group 1, and the opposite eyes (which were ipsilateral to the ICA with less than $70 \%$ stenosis) were included in Group 2. The two groups were compared in terms of the percentage of ICA stenosis, peak systolic velocity of ICA, SFCT measurements, intraocular pressure, and ocular perfusion pressure. In addition, the correlation between the percentage of ICA stenosis and the thickness of the subfoveal choroidal tissue was evaluated. The present study was performed according to the principles of the Declaration of Helsinki. Informed consent was obtained from each patient after a detailed explanation of the study. Patients were excluded if they met any of the following criteria: a history of previous retinal disease, media opacity that prevented OCT imaging, intraocular surgery within six months, glaucoma, high myopia with a refractive error greater than 6 diopters, or other eye diseases that could compromise the visual acuity. In addition, to avoid confounding by other known factors that can affect choroidal thickness, patients with uncontrolled hypertension (HT), as defined by a blood pressure > $140 / 90 \mathrm{mmHg}$ or the use of antihypertensive medication, and diabetes mellitus (DM) were excluded.

All patients underwent a baseline examination including refraction, best-corrected Snellen visual acuity, a dilated fundus and slit-lamp examination, and enhanced depth imaging optical coherence tomography (EDI-OCT). Detailed medical histories were obtained including HT, hypercholesterolemia, and smoking and history of cardiovascular or cerebrovascular disease. Smoking history was evaluated in pack years (1 pack year $=20$ cigarettes/day for one year).

Systolic and diastolic blood pressure were measured using an automated sphygmomanometer (BP-203 RVIIIB; Omron
Healthcare Co., Ltd., Kyoto, Japan), based on the cuff oscillometric method, with an appropriately sized cuff on the right arm in the sitting position after resting for $\geq 5$ minutes before the OCT measurement. The mean of 3 measurements was used for the analysis. The mean arterial blood pressure $(\mathrm{MABP})$ was calculated as mean arterial blood pressure $=$ $2 / 3$ diastolic blood pressure $+1 / 3$ systolic blood pressure. The mean ocular perfusion pressure was calculated as mean ocular perfusion pressure $=2 / 3$ mean arterial blood pressure - IOP.

2.1. Gray-Scale and Doppler US Examination. ICA stenosis was diagnosed using both the B-mode and Doppler ultrasound (Aplio MX, Toshiba Medical Systems, Corp., Otawara, Japan) methods with a $7.5 \mathrm{MHz}$ linear ultrasound probe by an experienced radiologist (SM). Colored Doppler US was performed both on and distal to the plaque, which was diagnosed by B-mode ultrasound with gray scale in the longitudinal plane. Spectral Doppler flow was measured within the areas that showed jet flow, heterogeneous color patterns, and abnormal flow of narrowed areas. A consensus of the panel was used to develop the criteria for the table of recommended Doppler thresholds for the diagnosis of ICA stenosis [24]. Both the degree of stenosis in the ICA and the Peak Systolic Velocity (PSV) were recorded.

2.2. OCT Protocol and Choroidal Thickness Measurement. All eyes were examined with the RTVue-100 OCT device (Optovue Inc., Fremont, CA) by the EDI-OCT technique. The RTVue-100 OCT device utilizes a choroid mode in which the OCT image is automatically inverted so that the chorioretinal interface is adjacent to zero delay. The line protocol of OCT was performed in this mode. The line protocol performed in the choroid mode included a horizontal single line image through the foveal center and was derived from an average of 16 frames (each frame consisted of $1024 \mathrm{~A}$-scans, and a total of 16,384 data points were averaged to a single scan image). The measurements were done in $1: 1$ microns mode. The subfoveal choroidal thickness measurements were performed manually using the caliper function of the OCT device. The SFCT was defined as a vertical line perpendicular to the center of the fovea from the outer portion of the hyperreflective line of Bruch's membrane to the choroidoscleral interface (Figures 1(a) and 1(b)). All OCT examinations were performed by the same experienced technician, and the SFCT measurements in the current study were performed by a retina specialist who was blinded to the group designations (CU).

2.3. Statistical Analysis. All data were analyzed using SPSS Version 18.0 (SPSS, Inc., Chicago, IL). Data are expressed as the means \pm standard deviation of the mean. The Kolmogorov-Smirnov test was used to identify the normality of distribution. Student's $t$-test was used to compare parameters between groups. Spearman rank correlation coefficient was used to evaluate associations between the measured variables. Degrees of agreement between examiners was assessed by calculating intraclass correlation coefficients (ICC) and 95\% confidence intervals (CI). Results with a $P$ value $<0.05$ were considered to be significant. 

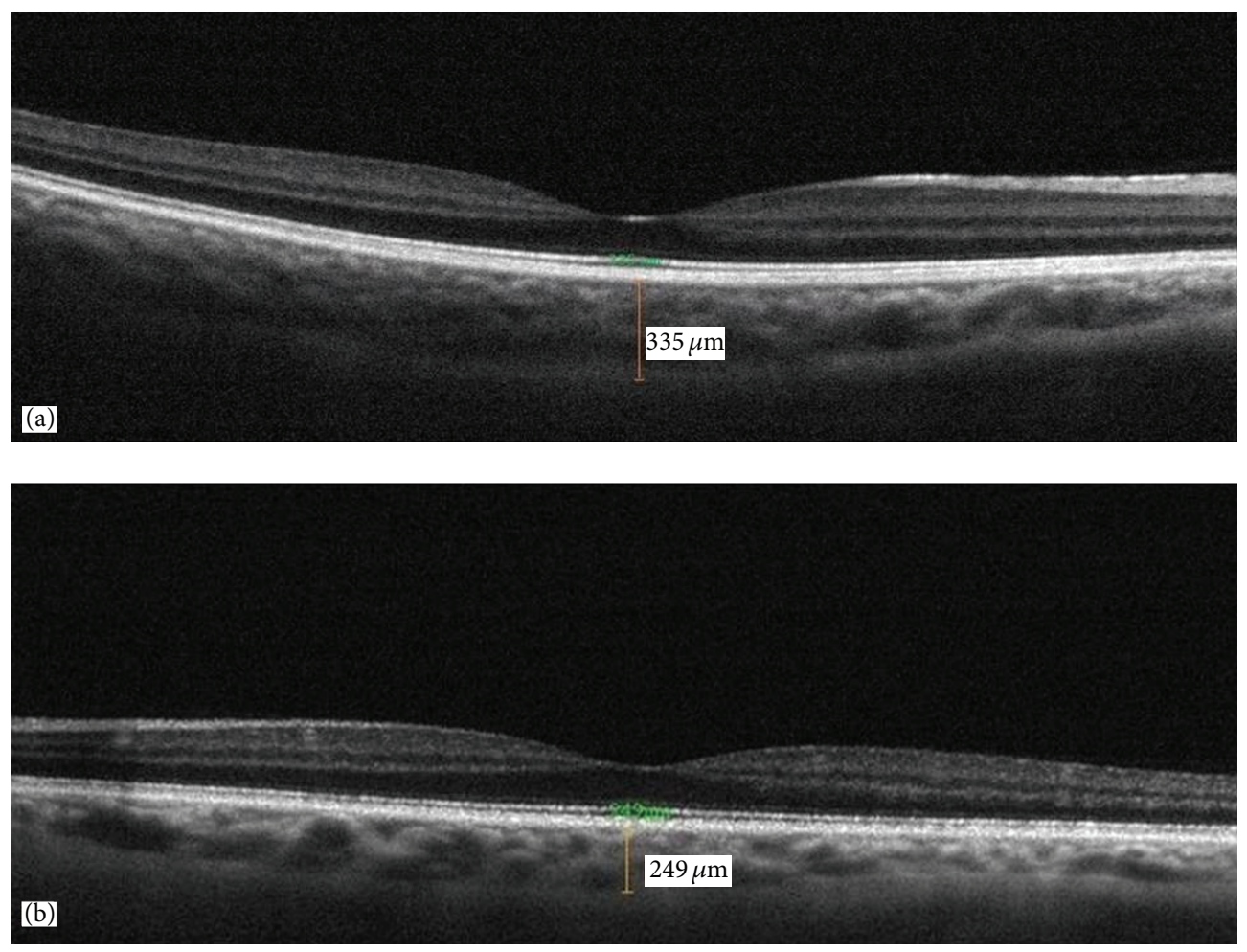

FIGURE 1: Sixty-four-year-old male patient: (a) $335 \mu \mathrm{m}$ subfoveal choroidal thickness in right eye (85\% stenosis in right internal carotid artery) and (b) $249 \mu \mathrm{m}$ subfoveal choroidal thickness in left eye (55\% stenosis in left internal carotid artery).

\section{Results}

Forty-two eyes of 21 patients (13 males and 8 females) with more than $70 \%$ carotid artery stenosis on one side and less than $70 \%$ stenosis on the other side were recruited for the study. The mean age of the patients was $71.9 \pm 10.8$ years (with a range of $52-84$ years). The mean ICA stenosis was $60.7 \pm 14.6 \mu \mathrm{m}$ for all of the patients. The characteristics and demographic features of the patients are shown in Table 1.

There was a statistically significant difference in the mean SFCT value between Group $1(231.9 \pm 44.6 \mu \mathrm{m})$ and Group 2 $(216.2 \pm 46.8 \mu \mathrm{m})(P=0.028)$. However, the refractive error and mean IOP and OPP values were not significantly different between the two groups $(P=0.530, P=0.546$, and $P=$ 0.430 , resp.). Table 2 shows the subfoveal choroidal thickness and other clinical measurements of the two groups.

There was a statistically significant positive correlation between the percentage of internal carotid artery stenosis and SFCT $(r=0.896, P=0.001)$. This was shown in scatter plot with regression line (Figure 2). However, the SFCT value was not significantly associated with the IOP or OPP $(r=0.089$, $P=0.693$ and $r=0.009, P=0.726$, resp.).

A correlation analysis between the SFCT and the percentage of ICA stenosis, IOP, and OPP of the whole cohort is shown in Table 3.

The mean SFCT measurements in the current study were performed by two independent examiners BS [Examiner 1] and CU [Examiner 2]. The mean subfoveal choroidal thicknesses measured by examiner 1 and examiner 2 were $223.5 \pm$
TABLE 1: The demographic features and baseline characteristics of patients.

\begin{tabular}{lc}
\hline Number of patients $(n)$ & 21 \\
Age $($ mean \pm SD) & $71.9 \pm 10.8(52-84)$ \\
Sex, male/female & $13 / 8$ \\
Hypertension $(n, \%)$ & $21(100 \%)$ \\
Hypercholesterolemia $(n, \%)$ & $21(100 \%)$ \\
Coronary artery disease $(n, \%)$ & $16(76.1 \%)$ \\
Tobacco use $(n, \%)$ & $18(85.7 \%)$ \\
Sleep apnea syndrome $(n, \%)$ & $9(42.8 \%)$ \\
History of stroke $(n, \%)$ & $12(57.1 \%)$ \\
SBP, mmHg [mean \pm SD (range)] & $104 \pm 1.5(90-120)$ \\
DBP, mmHg [mean \pm SD (range) & $81.3 \pm 1.3(65-90)$ \\
MABP, mmHg [mean \pm SD (range) & $86.3 \pm 1.1(70-95)$ \\
\hline
\end{tabular}

SBP: systolic blood pressure; DBP: diastolic blood pressure; MABP: mean arterial blood pressure; and SD: standard deviation.

$46.8 \mu \mathrm{m}$ and $226.2 \pm 47.1 \mu \mathrm{m}$, respectively. The level of agreement between examiner 1 and examiner 2 was $96 \%$ (ICC: $0.969 ; 95 \%$ CI: $0.956-0.982 ; P<0.01)$.

\section{Discussion}

The ophthalmic artery, which is the first branch of the internal carotid artery, branches to form the central retinal artery and the posterior ciliary arteries. In $90 \%$ of eyes, the posterior 
TABLE 2: Subfoveal choroidal thickness and other clinical measurements in both groups.

\begin{tabular}{lccc}
\hline & Group 1 & Group 2 & $P$ value \\
\hline SFCT, mean \pm SD & $231.9 \pm 44.6$ & $216.2 \pm 46.8$ & $P=0.028$ \\
Percentage of ICA stenosis (\%), mean \pm SD & $74 \pm 4.9(70-85)$ & $47.5 \pm 7.7(35-60)$ & \\
PSV (cm/sec), mean \pm SD & $235.53 \pm 7.8$ & $118.95 \pm 16.1$ & \\
Refractive error [mean \pm SD (diopters)] & $-0.27 \pm 1.6$ & $-0.34 \pm 1.8$ & \\
IOP mmHg [mean \pm SD, (range)] & $15.2 \pm 1.1(12-18)$ & $14.9 \pm 18(11-17)$ & $P=0.530$ \\
OPP mmHg [mean \pm SD, (range)] & $41.8 \pm 1.6$ & $40.9 \pm 1.3$ & $P=0.546$ \\
\hline
\end{tabular}

ICA: internal carotid artery; SFCT: subfoveal choroidal thickness; PSV: peak systolic velocity; OPP: ocular perfusion pressure; IOP: intraocular pressure; and SD, standard deviation; $P$ value of less than 0.05 was considered to be significant.

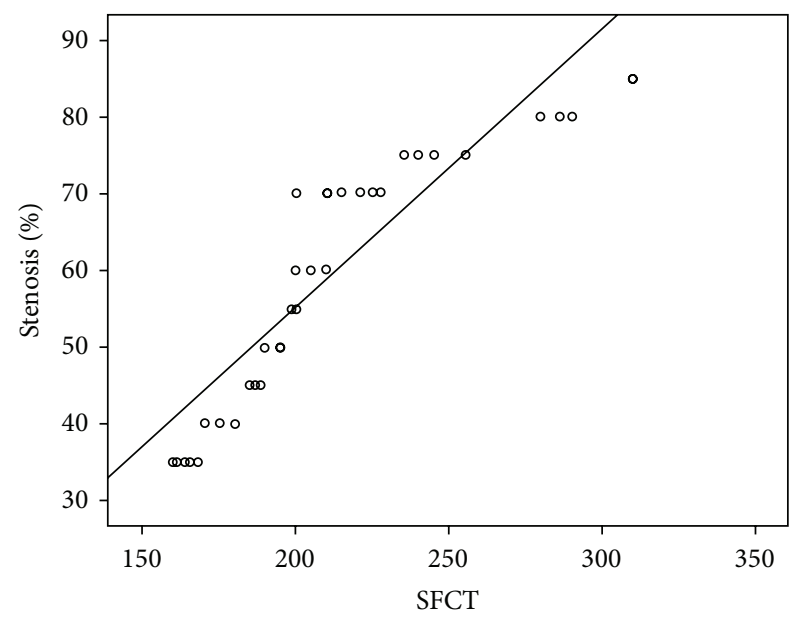

FIGURE 2: Scatter plot graph showing the relationship percentage of internal carotid artery stenosis and subfoveal choroidal thickness (SFCT).

TABLE 3: Spearman rank correlation analyses between the subfoveal choroidal thickness and the percentage of ICA stenosis, IOP, and OPP.

\begin{tabular}{lcc}
\hline & SFCT & \\
& Spearman rank correlation & \\
\hline Percentage of ICA stenosis & $r=0.893$ & $P=0.001$ \\
IOP & $r=0.089$ & $P=0.693$ \\
OPP & $r=0.009$ & $P=0.726$ \\
\hline
\end{tabular}

SFCT: subfoveal choroidal thickness; ICA: internal carotid artery; OPP: ocular perfusion pressure; and IOP: intraocular pressure; $P$ value of less than 0.05 was considered to be significant.

choroid receives its blood supply from two major posterior ciliary arteries: the medial and lateral posterior ciliary arteries. The long posterior ciliary arteries supply blood to the anterior uvea. Smaller short posterior ciliary arteries pass to the posterior part of the eyeball around the optic nerve and supply choroid and ciliary processes [3].

When the ICA is obstructed, blood flow to the brain is maintained by the opening of collateral channels between the terminal external carotid artery branches and the terminal branches of the ophthalmic artery. The opening up of ECAICA collateral channels via the ophthalmic artery can cause many signs and symptoms such as delay/asymmetry of ECA pulses, angular, brow, cheek pulses, frontal artery sign, dilated episcleral arteries, and Olivarius' external carotid sign. Retrograde flow to the ICA occurs through these channels via the ophthalmic artery, resulting in hypoperfusion of the retinal and choroidal circulation [25]. This phenomenon may lead to compromised choroidal flow and result in photoreceptor dysfunction. Structurally and functionally, normal choroidal vasculature is crucial for proper retinal function. Numerous prospective, randomized, and multicenter studies have been designed to evaluate choroidal thickness in normal and disease states [15-23]. However, no clear evidence has been found regarding the effect of internal carotid artery stenosis on choroidal thickness.

The present study was designed to evaluate whether ICA stenosis of more than $70 \%$ has an effect on ipsilateral subfoveal choroidal thickness compared to the opposite eye. Our data indicate that the SFCT is significantly thicker in eyes on the side with more than $70 \%$ stenosis $(231.9 \pm 44.6$ versus $216.2 \pm 46.8, P=0.028)$.

When stenosis occludes at least $70 \%$ of the lumen, or if the residual lumen diameter is $\leq 1.5 \mathrm{~mm}$, a carotid bruit is usually heard at the origin of the ICA [26]. One of the most fundamental principles of the circulation is the ability to control its own blood flow in proportion to its metabolic requirements. Long term control of local blood flow means slow changes over a period of time resulting in change in physical size and number of the blood vessels. One of the most important mechanisms of the long term regulation is the change in the vascularity, as if when the metabolism of a given tissue increases vascularity increases. Angiogenesis is also an important local blood control mechanism in order to protect tissues from ischemia. Inadequate blood supply leads to release of the angiogenic factors which further leads to promoting new vessel formation. Development of collateral formation is also an important long term blood flow regulation [27]. Concerning the high metabolic demand of the choroid, increased SFCT that we observed at the stenosis site might be a dilatation in the choriocapillaris vasculature to prevent retinal and choroidal ischemia as a result of the diminished blood flow due to ICA stenosis.

In accordance with our results, Salazar et al. investigated alterations in the choroid in hypercholesterolemic rabbits and concluded that the choroid was thicker in the presence of hypercholesterolemia [28]. Similarly, Wong et al. reported that the mean SFCT was thicker among patients with hypercholesterolemia [29]. Altinkaynak et al. evaluated the SFCT 
measured by EDI-OCT in the eyes of chronic heart failure (CHF) patients. In contrast to our results, they found that the mean SFCT was $181.2 \pm 80.23 \mu \mathrm{m}$ in the study group and $283.6 \pm 52.4 \mu \mathrm{m}$ in the control group, indicating that the SFCT is lower in the eyes of CHF patients compared to age- and gender-matched control subjects. A compensatory peripheral vasoconstriction develops in $\mathrm{CHF}$ in order to maintain satisfactory blood pressure and vasoconstriction in orbital and choroidal vessels causes a lower SFCT in CHF patients [30].

Physiological factors may have an effect on choroidal thickness [29]. Aging is one of the most important factors associated with changes in choroidal thickness. In our study, the mean SFCT of all eyes was $216.2 \pm 46.8 \mu \mathrm{m}$. Several studies have reported mean SFCTs between $261 \pm 88 \mu \mathrm{m}$ and $354 \pm$ $111 \mu \mathrm{m}$ in different age groups [31-33]. Chen et al. evaluated the interocular symmetry of macular choroidal thickness using enhanced depth imaging optical coherence tomography in 100 eyes of 50 healthy subjects [34]. They concluded that there was no significant difference in the intraindividual choroidal thickness.

In our study, there was a statistically significant positive correlation between the percentage of internal carotid artery stenosis and SFCT $(r=0.44, P=0.03)$, but there was no significant correlation between SFCT and IOP and OPP, indicating that the subfoveal choroidal thickness increases as the percentage of stenosis increases. Recent studies have already indicated that the thickness of the choroid changes in the course of some disorders such as the glaucoma. Kubota et al. revealed decreased choroidal thickness in eyes with secondary angle-closure glaucoma [35]; Arora et al. reported that choroidal thickness was significantly greater in the angleclosure glaucoma group than in the open angle glaucoma group and normal subjects, with no significant difference between eyes with open angle glaucoma and normal subjects [36]. Hosseini and colleagues revealed that, except for the temporal region, choroidal thickness did not differ between glaucomatous and control eyes [37]. A recent study conducted by Wei et al. investigated the association between choroidal thickness and IOP and OPP values, and similar to our results, no statistically significant correlation was found in healthy subjects [38]. Altinkaynak et al. also evaluated the correlation between choroidal thickness and IOP and OPP values in congestive heart failure (CHF) patients, and they found no significant correlation between SFCT and IOP and OPP in CHF patients [30].

In conclusion, subfoveal choroidal thickness was found to be high in the presence of more than 70\% ICA stenosis in the current study. Spectral-domain OCT is a useful, noninvasive, diagnostic tool for the assessment of the choroid and the evaluation of chorioretinal vascular changes in an aging population with various atherosclerotic risk factors. Further studies with larger cohorts and longer follow-up times are needed to evaluate the effects of various atherosclerotic risk factors on choroidal thickness.

\section{Conflict of Interests}

The authors have no conflict of interests to declare.

\section{Acknowledgment}

This study was edited by American Journal Experts, Certificate Verification Key: 9225-22A4-C9D0-DBFF-094B.

\section{References}

[1] L. M. Parver, C. Auker, and D. O. Carpenter, "Choroidal blood flow as a heat dissipating mechanism in the macula," American Journal of Ophthalmology, vol. 89, no. 5, pp. 641-646, 1980.

[2] S. S. Hayreh, "Acute retinal arterial occlusive disorders," Progress in Retinal and Eye Research, vol. 30, no. 5, pp. 359-394, 2011.

[3] S. S. Hayreh, "Posterior ciliary artery circulation in health and disease: the Weisenfeld lecture," Investigative Ophthalmology and Visual Science, vol. 45, no. 3, pp. 749-757, 2004.

[4] D. L. Nickla and J. Wallman, "The multifunctional choroid," Progress in Retinal and Eye Research, vol. 29, no. 2, pp. 144-168, 2010.

[5] R. F. Spaide, H. Koizumi, and M. C. Pozonni, "Enhanced depth imaging spectral-domain optical coherence tomography," American Journal of Ophthalmology, vol. 146, no. 4, pp. 496-500, 2008.

[6] G. M. Fox, A. Sivalingham, and G. C. Brown, "Ocular ischemic syndrome," in Ophthalmology, M. Yanoff and J. Duker, Eds., Mosby, St Louis, Miss, USA, 1999.

[7] J. B. Mizener, P. Podhajsky, and S. S. Hayreh, "Ocular ischemic syndrome," Ophthalmology, vol. 104, no. 5, pp. 859-864, 1997.

[8] J. D. Dugan Jr. and W. R. Green, "Ophthalmologic manifestations of carotid occlusive disease," Eye, vol. 5, no. 2, pp. 226-238, 1991.

[9] T. P. Kearns and R. W. Hollenhorst, "Venous-stasis retinopathy of occlusive disease of the carotid artery," Mayo Clinic Proceedings, vol. 38, pp. 304-312, 1963.

[10] T. P. Kearns, R. G. Siekert, and T. M. Sundt Jr., "The ocular aspects of bypass surgery of the carotid artery," Mayo Clinic Proceedings, vol. 54, no. 1, pp. 3-11, 1979.

[11] N. J. C. Sarkies, J. S. Shilling, and R. W. Ross Russell, "Fluorescein angiography in carotid disease," Transactions of the Ophthalmological Societies of the United Kingdom, vol. 105, no. 4, pp. 489-493, 1986.

[12] P. Kersemakers, M. Beintema, and J. Lodder, "Venous stasis retinopathy unlikely results from internal carotid artery obstruction alone," Cerebrovascular Diseases, vol. 2, no. 5, pp. 305-307, 1992.

[13] M. De Weerd, J. P. Greving, A. W. F. de Jong, E. Buskens, and M. L. Bots, "Prevalence of asymptomatic carotid artery stenosis according to age and sex: systematic review and metaregression analysis," Stroke, vol. 40, no. 4, pp. 1105-1113, 2009.

[14] H.-H. Eckstein, A. Kühnl, A. Dörfler, I. B. Kopp, H. Lawall, and P. A. Ringleb, "The diagnosis, treatment and follow-up of extracranial carotid stenosis: a multidisciplinary GermanAustrian Guideline Based on Evidence and Consensus," Deutsches Arzteblatt International, vol. 110, no. 27-28, pp. 468476, 2013.

[15] S. E. Chung, S. W. Kang, J. H. Lee, and Y. T. Kim, "Choroidal thickness in polypoidal choroidal vasculopathy and exudative age-related macular degeneration," Ophthalmology, vol. 118, no. 5, pp. 840-845, 2011.

[16] I. Maruko, T. Iida, Y. Sugano et al., "Subfoveal choroidal thickness after treatment of Vogt-Koyanagi-Harada disease," Retina, vol. 31, no. 3, pp. 510-517, 2011. 
[17] J. Yeoh, W. Rahman, F. Chen et al., "Choroidal imaging in inherited retinal disease using the technique of enhanced depth imaging optical coherence tomography," Graefe's Archive for Clinical and Experimental Ophthalmology, vol. 248, no. 12, pp. 1719-1728, 2010.

[18] W. Rahman, F. K. Chen, J. Yeoh, P. Patel, A. Tufail, and L. Da Cruz, "Repeatability of manual subfoveal choroidal thickness measurements in healthy subjects using the technique of enhanced depth imaging optical coherence tomography," Investigative Ophthalmology \& Visual Science, vol. 52, no. 5, pp. 2267-2271, 2011.

[19] I. Maruko, T. Iida, Y. Sugano, M. Saito, and T. Sekiryu, "Subfoveal retinal and choroidal thickness after verteporfin photodynamic therapy for polypoidal choroidal vasculopathy," American Journal of Ophthalmology, vol. 151, no. 4, pp. 594-603, 2011.

[20] M. Hirata, A. Tsujikawa, A. Matsumoto et al., "Macular choroidal thickness and volume in normal subjects measured by swept-source optical coherence tomography," Investigative Ophthalmology and Visual Science, vol. 52, no. 8, pp. 4971-4978, 2011.

[21] E. J. Sigler, J. C. Randolph, J. I. Calzada, and S. Charles, "Smoking and choroidal thickness in patients over 65 with early-atrophic age-related macular degeneration and normals," Eye, vol. 28, no. 7, pp. 838-846, 2014.

[22] A. Boltz, A. Luksch, B. Wimpissinger et al., "Choroidal blood flow and progression of age-related macular degeneration in the fellow eye in patients with unilateral choroidal neovascularization," Investigative Ophthalmology and Visual Science, vol. 51, no. 8, pp. 4220-4225, 2010.

[23] J. E. Grunwald, A. M. Maguire, and J. Dupont, "Retinal hemodynamics in retinitis pigmentosa," American Journal of Ophthalmology, vol. 122, no. 4, pp. 502-508, 1996.

[24] E. G. Grant, C. B. Benson, G. L. Moneta et al., "Carotid artery stenosis: gray-scale and Doppler US diagnosis-Society of Radiologists in Ultrasound Consensus Conference," Radiology, vol. 229, no. 2, pp. 340-346, 2003.

[25] A. Arthur, A. Alexander, S. Bal, A. Sivadasan, and S. Aaron, "Ophthalmic masquerades of the atherosclerotic carotids," Indian Journal of Ophthalmology, vol. 62, no. 4, pp. 472-476, 2014.

[26] L. R. Caplan, Caplan's Stroke, A Clinical Approach, Saunders, Philadelphia, Pa, USA, 4th edition, 2009.

[27] A. C. Guyton and J. E. Hall, Guyton and Hall Textbook of Medical Physiology, Saunders, Philadelphia, Pa, USA, 9th edition, 1996.

[28] J. J. Salazar, A. I. Ramírez, R. de Hoz et al., "Alterations in the choroid in hypercholesterolemic rabbits: reversibility after normalization of cholesterol levels," Experimental Eye Research, vol. 84, no. 3, pp. 412-422, 2007.

[29] I. Y. Wong, R. L. Wong, P. Zhao, and W. W. Lai, "Choroidal thickness in relation to hypercholesterolemia on enhanced depth imaging optical coherence tomography," Retina, vol. 33, no. 2, pp. 423-428, 2013.

[30] H. Altinkaynak, N. Kara, N. Sayin, H. Güneş, S. Avsąr, and A. T. Yazıc1, "Subfoveal choroidal thickness in patients with chronic heart failure analyzed by spectral-domain optical coherence tomography," Current Eye Research, vol. 39, no. 11, pp. 1123-1128, 2014.

[31] Y. Ikuno, K. Kawaguchi, T. Nouchi, and Y. Yasuno, "Choroidal thickness in healthy Japanese subjects," Investigative Ophthalmology and Visual Science, vol. 51, no. 4, pp. 2173-2176, 2010.

[32] R. Margolis and R. F. Spaide, "A pilot study of enhanced depth imaging optical coherence tomography of the choroid in normal eyes," American Journal of Ophthalmology, vol. 147, no. 5, pp. 811-815, 2009.

[33] X. Ding, J. Li, J. Zeng et al., "Choroidal thickness in healthy Chinese subjects," Investigative Opthalmology \& Visual Science, vol. 52, no. 13, pp. 9555-9560, 2011.

[34] F. K. Chen, J. Yeoh, W. Rahman, P. J. Patel, A. Tufail, and L. Da Cruz, "Topographic variation and interocular symmetry of macular choroidal thickness using enhanced depth imaging optical coherence tomography," Investigative Ophthalmology and Visual Science, vol. 53, no. 2, pp. 975-985, 2012.

[35] T. Kubota, J. B. Jonas, and G. O. H. Naumann, "Decreased choroidal thickness in eyes with secondary angle closure glaucoma. An aetiological factor for deep retinal changes in glaucoma?" British Journal of Ophthalmology, vol. 77, no. 7, pp. 430432, 1993.

[36] K. S. Arora, J. L. Jefferys, E. A. Maul, and H. A. Quigley, "The choroid is thicker in angle closure than in open angle and control eyes," Investigative Ophthalmology \& Visual Science, vol. 53, no. 12, pp. 7813-7818, 2012.

[37] H. Hosseini, N. Nilforushan, S. Moghimi et al., "Peripapillary and macular choroidal thickness in glaucoma," Journal of Ophthalmic and Vision Research, vol. 9, no. 2, pp. 154-161, 2014.

[38] W. B. Wei, L. Xu, J. B. Jonas et al., "Subfoveal choroidal thickness: the Beijing eye study," Ophthalmology, vol. 120, no. 1, pp. 175180, 2013. 


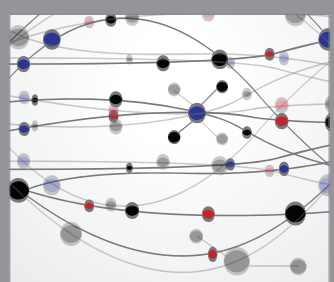

The Scientific World Journal
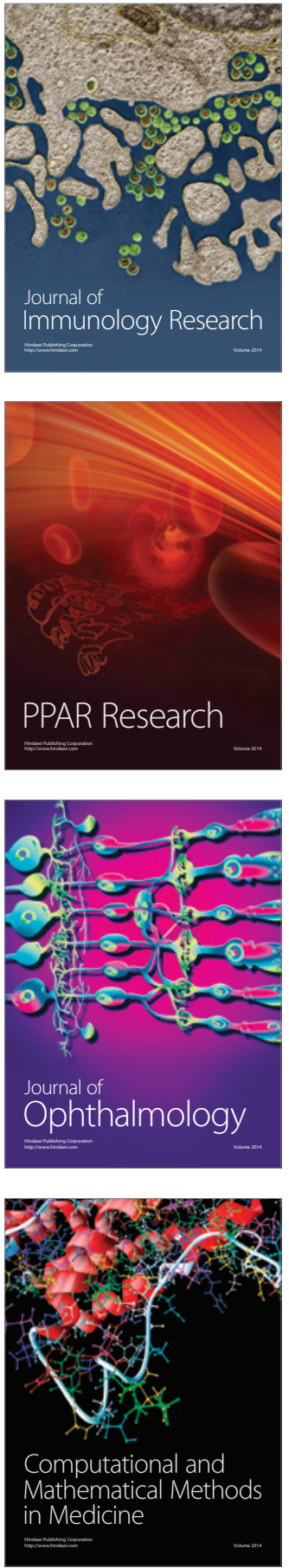

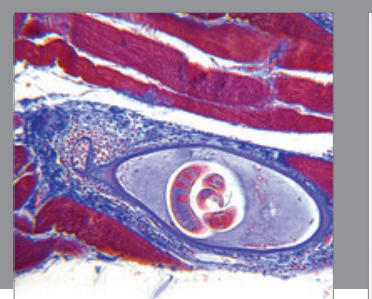

Gastroenterology Research and Practice

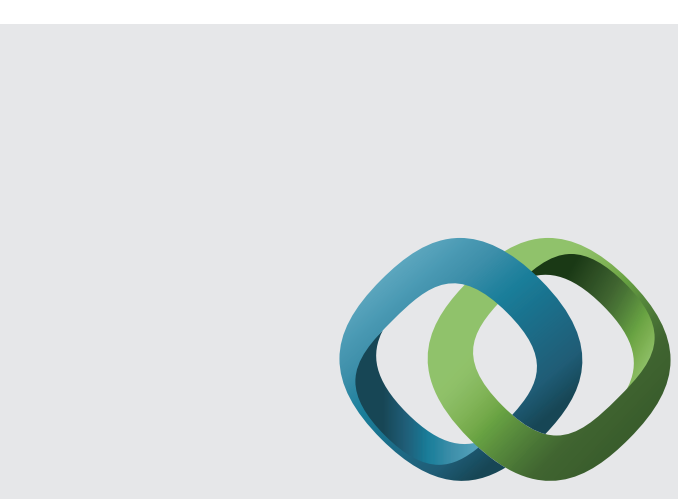

\section{Hindawi}

Submit your manuscripts at

http://www.hindawi.com
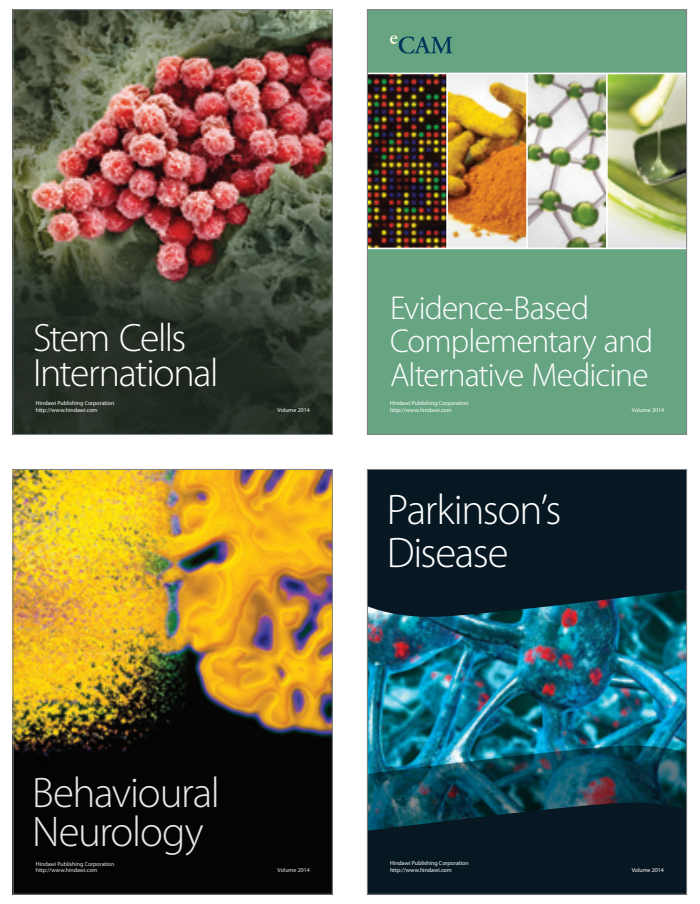
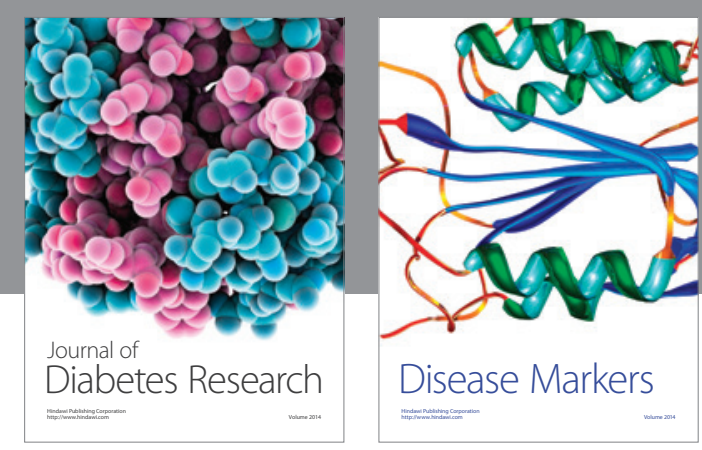

Disease Markers
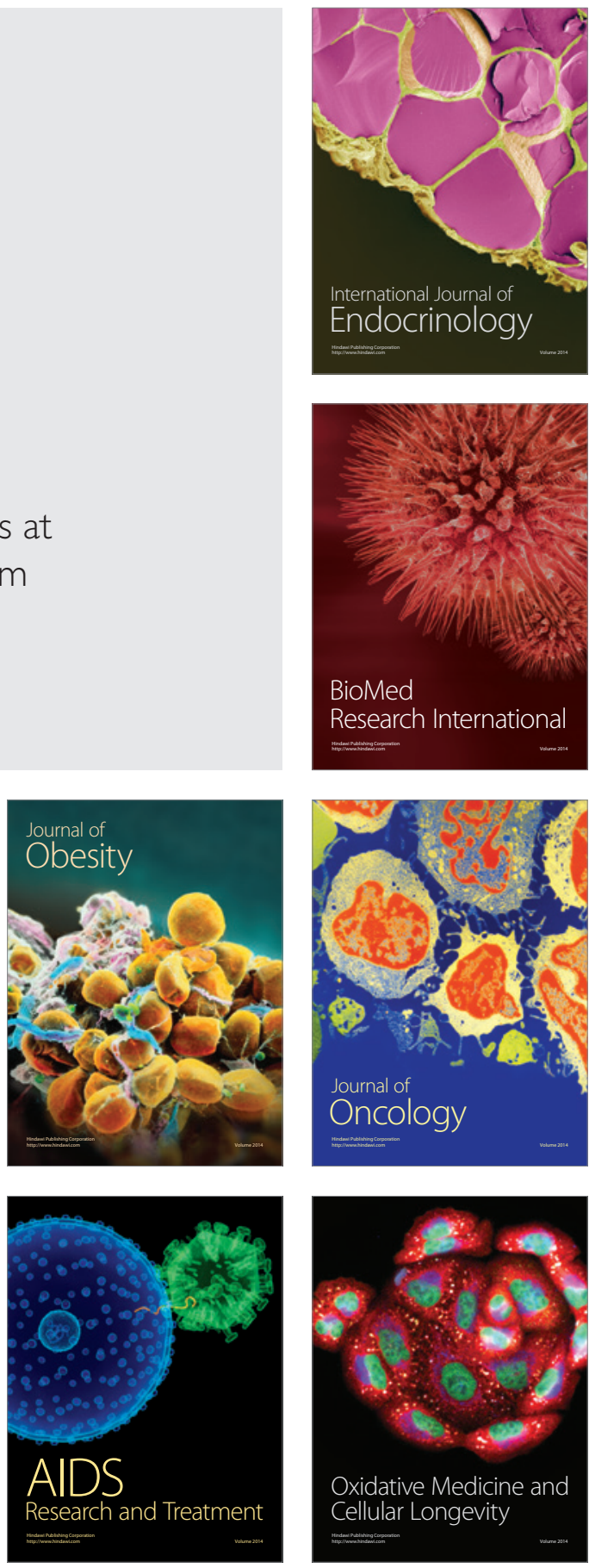